\title{
Fatores associados e indicações para a prática de cesariana: um estudo caso-controlo
}

Ana Rita Oliveira*

\section{RESUMO}

Objetivos: Determinar se são fatores associados à realização de cesarianas: nível de escolaridade, índice de massa corporal (IMC), hábitos tabágicos maternos, nuliparidade, antecedentes de cesariana prévia, setor da vigilância obstétrica, macrossomia e antecedentes de diabetes ou hipertensão arterial (HTA); Analisar: i) a associação entre a prática de cesarianas e complicações pós-parto (hemorragia, infeção ou necessidade de reinternamento); ii) a associação entre a prática de cesarianas e a taxa de aleitamento materno exclusivo à data da alta; iii) descrever as indicações apontadas para a realização de cesariana.

Tipo de estudo: Analítico retrospetivo tipo caso-controlo.

Local: Hospital Santo André, Leiria.

População: Amostra aleatória estratificada não proporcional de 400 mulheres (200 submetidas a cesariana e 200 a parto vaginal) cujo parto decorreu no ano de 2010.

Métodos: Pesquisa de associação entre as variáveis e o tipo de parto por análise estatística bivariada, com cálculo do odds-ratio e teste do qui-quadrado, adotando-se um nível de significância de 0,05.

Resultados: A percentagem de realização de cesarianas aumentou com a escolaridade $(p<0,001)$. No grupo das cesarianas a taxa de nuliparidade foi de $57,0 \%(p=0,002)$. Das multíparas submetidas a cesariana, $87,7 \%$ apresentavam antecedentes de cesariana $(p<0,001)$. Entre aquelas submetidas a cesariana, verificou-se que a maior prevalência de HTA $(72,4 \%, p=0,012)$ e de vigilância obstétrica ocorreu no setor privado $(56,9 \%, p=0,002)$. Em 81,0\% dos recém-nascidos sob aleitamento artificial e $58,5 \%$ dos que faziam aleitamento misto, à data da alta, o parto ocorreu por cesariana $(p=0,005)$. Dos partos complicados por infeção $88,9 \%$ eram cesariana $(p=0,001)$. Não foi encontrada associação estatisticamente significativa entre a realização de cesariana e hábitos tabágicos, IMC materno, diabetes e macrossomia.

Conclusões: Maior escolaridade, nuliparidade, vigilância obstétrica no setor privado, cesariana prévia, HTA, aleitamento artificial e infeção pós-parto associaram-se à prática de cesariana.

Palavras-chave: Cesariana; Parto; Prevalência.

\section{INTRODUÇÃO}

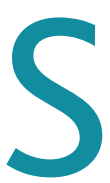

egundo a Organização Mundial de Saúde (OMS), os cuidados perinatais visam a manutenção da saúde materno-fetal através da mínima intervenção médica. ${ }^{1}$ Assim, a cesariana é um procedimento cirúrgico desenvolvido com o intuito de reduzir o risco de complicações maternas e fetais durante a gravidez e o parto que, dado não ser inócuo e acarretar implicações e riscos para o futuro reprodutivo da parturiente, não deve ser levado a cabo na ausência das indicações médicas descritas. ${ }^{2}$

*Interna da formação específica em Medicina Geral e Familiar USF Santiago, Leiria
Estudos revelam que a realização de cesarianas primárias eletivas terá implicações no aumento da morbilidade materna (hemorragia no pós-parto, infeção e tromboembolismo), acarretando um risco até três vezes maior de mortalidade materna e aumentando o risco de placenta prévia, placenta acreta, rotura uterina e histerectomia periparto, em gravidezes subsequentes. ${ }^{4}$ Verifica-se, ainda, que em mulheres submetidas a cesariana a probabilidade de início e manutenção do aleitamento materno exclusivo é inferior. ${ }^{5}$

Deste modo, a OMS considera injustificável a existência de taxas de cesarianas superiores a $15 \%$, pois existe evidência que taxas superiores não estão asso- 
ciadas a redução adicional da morbi-mortalidade materno-fetal., ${ }^{2,3}$ No entanto, tem-se assistido a um aumento consecutivo e sustentado das taxas de cesariana a nível mundial, tanto em países desenvolvidos (Estados Unidos, Inglaterra, Itália, Portugal, Austrália, China) como naqueles em vias de desenvolvimento (Brasil). ${ }^{2,6,7,8,9}$

Nos Estados Unidos da América, as cesarianas apresentam-se como o procedimento cirúrgico mais comum entre indivíduos do sexo feminino. Após um declínio nas taxas de partos por cesariana, entre $1991 \mathrm{e}$ 1996, devido ao aumento de partos vaginais em mulheres com antecedentes de parto por cesariana, resultado de medidas governamentais, assiste-se desde então a um aumento abrupto no número de cesarianas, tendo-se atingido em 2007 uma taxa de 31,8\%. ${ }^{2}$

Dados oficiais relativamente à taxa global de cesarianas em Portugal revelam que, em 2007, a taxa de parto por cesariana alcançou os $34,8 \% .{ }^{10}$ Valores alusivos apenas aos hospitais públicos nacionais apontam para uma taxa de cesarianas em 2007 de 32,4\%, em 2008 de $32,6 \%$ e em 2009 de $33,2 \% .{ }^{10}$ Dada a tendência ligeiramente crescente da taxa de cesarianas nos hospitais públicos portugueses, juntamente com o aumento do número de partos em hospitais privados, onde a taxa global de cesarianas em 2005 atingiu os $65,9 \%,{ }^{10}$ estima-se que a taxa global de cesarianas em 2009 tenha rondado os $36 \% .{ }^{10}$ Portugal é, assim, um dos países europeus com maior taxa de cesarianas, tendo sido ultrapassado em 2007 apenas pela Itália. ${ }^{10}$ Todos os restantes países europeus têm uma taxa de cesarianas que não ultrapassa os $30 \%$ e vários têm uma taxa inferior a $20 \% .^{10}$ A causa deste aumento é multifatorial, prendendo-se não só com fatores sociodemográficos, culturais e económicos associados à preferência materna, mas também com aspetos relacionados com os sistemas de saúde no âmbito da prática médica, das preferências médicas, das preocupações médico-legais e dos interesses económicos dos vários intervenientes no processo., ${ }^{2,11}$

No Hospital Santo André, em Leiria, Portugal, durante o ano de 2010, a taxa de cesarianas foi de $31,8 \%$ (fonte: programa informático «Bloco de Partos®»).

Este estudo tem como objetivo principal determinar se o nível de escolaridade, o índice de massa corporal (IMC), os hábitos tabágicos maternos, a nuliparidade, os antecedentes de cesariana prévia, a vigilân- cia no setor privado, a macrossomia e os antecedentes de diabetes ou hipertensão arterial (HTA) estão associados à realização/prática de cesariana.

Pretende ainda, como objetivos secundários: i) analisar a associação entre a prática de cesarianas e as complicações no pós-parto (hemorragia, infeção pós-parto ou necessidade de reinternamento); ii) analisar a associação entre a prática de cesarianas e a taxa de aleitamento materno exclusivo à data da alta; iii) descrever as indicações apontadas para a realização de cesariana.

\section{MÉTODOS}

Estudo caso-controlo, observacional, analítico, retrospetivo. A população do estudo foram todas as mulheres com partos ocorridos no Hospital Santo André no ano civil de 2010.

A recolha de dados, que decorreu de 3 de outubro a 30 de novembro de 2011, foi realizada por um único investigador, a autora do estudo, através da consulta dos processos clínicos, não informatizados, das utentes.

Foram incluídas no estudo todas as utentes cujo parto decorreu no Hospital Santo André, durante o ano civil de 2010. Excluíram-se as utentes cujos processos clínicos fossem ilegíveis ou apresentassem o preenchimento insuficiente das variáveis estudadas (mais de 3 parâmetros não preenchidos).

O tamanho da amostra dos casos (cesarianas) foi calculado através da utilização da ferramenta sample size calculator (acedida online em http://www.surveysystem.com/sscalc.htm), para um nível de confiança de $95 \%$, uma margem de erro de $5 \%$, tendo tido por base o número de partos decorridos no Hospital Santo André no referido ano $(n=2.294)$ e a percentagem de cesarianas no referido ano $(38,1 \%)$, valor consultado no programa informático «Bloco de Partos®». De acordo com este cálculo, deveria ter sido estudada uma amostra de 252 cesarianas.

Por limitação de recursos, selecionaram-se 400 processos, 200 de partos por cesariana (casos) e 200 de partos vaginais (controlos), por amostragem aleatória estratificada, sem reposição, não proporcional, de 3 em 3 processos para as cesarianas e de $6 \mathrm{em} 6$ processos para os partos vaginais espontâneos.

Foram analisadas as seguintes variáveis: sociodemográficas (idade, escolaridade materna); IMC; historial obstétrico presente e pregresso (hábitos tabágicos, 
paridade e, no caso das multíparas, o tipo de parto prévio, local de vigilância da gravidez - setor público versus privado - e idade gestacional); morbilidades obstétricas (diabetes, HTA); justificações apresentadas para a realização de cesariana; peso do recém-nascido; complicações no pós-parto (hemorragia, infeção e história de reinternamento) e tipo de aleitamento à data da alta (aleitamento materno exclusivo, aleitamento artificial ou aleitamento misto).

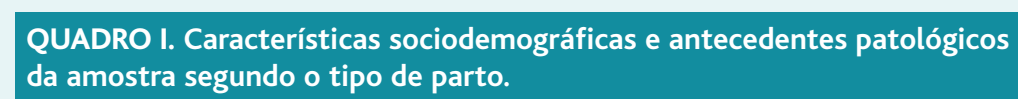

\begin{tabular}{|c|c|c|c|}
\hline Parâmetros avaliados & $\begin{array}{c}\text { Parto vaginal } \\
\text { n (\%) }\end{array}$ & $\begin{array}{c}\text { Parto cesariana } \\
\text { n (\%) }\end{array}$ & $\begin{array}{c}\text { P-value } \\
\text { (Qui-quadrado) }\end{array}$ \\
\hline $\begin{array}{l}\text { Idade materna } \\
\text { - } 15-19 \text { anos } \\
\text { - } 20-24 \text { anos } \\
\text { - } 25-29 \text { anos } \\
\text { - } 30-34 \text { anos } \\
\text { - } \geq 35 \text { anos }\end{array}$ & $\begin{array}{r}3(50,0) \\
33(67,3) \\
53(47,7) \\
67(44,7) \\
44(52,4)\end{array}$ & $\begin{array}{r}3(50,0) \\
16(32,7) \\
58(52,3) \\
83(55,3) \\
40(47,6)\end{array}$ & 0,091 \\
\hline 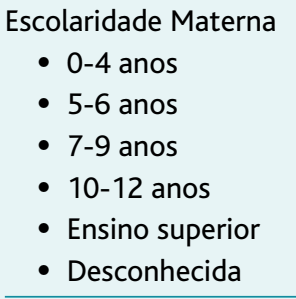 & $\begin{array}{r}0(0,0) \\
19(82,6) \\
45(68,2) \\
84(47,7) \\
46(39,7) \\
6(35,3)\end{array}$ & $\begin{array}{r}2(100,0) \\
4(17,4) \\
21(31,8) \\
92(52,3) \\
70(60,3) \\
11(64,7)\end{array}$ & $<0,001$ \\
\hline $\begin{array}{l}\text { IMC materno } \\
\begin{aligned} & \text { - }<25 \\
& \text { - } 25-29 \mathrm{~kg} / \mathrm{m} 2 \\
& \text { - } 30-34 \mathrm{~kg} / \mathrm{m} 2 \\
& \text { - }>35 \mathrm{~kg} / \mathrm{m} 2 \\
& \text { - } \text { Desconhecido }\end{aligned}\end{array}$ & $\begin{array}{r}75(45,5) \\
79(56,0) \\
27(50,0) \\
18(56,3) \\
1(12,5)\end{array}$ & $\begin{array}{r}90(54,5) \\
62(44,0) \\
27(50,0) \\
14(43,8) \\
7(87,5)\end{array}$ & 0,078 \\
\hline $\begin{array}{l}\text { Hábitos Tabágicos } \\
\text { - Sim } \\
\text { - Não }\end{array}$ & $\begin{array}{r}24(44,4) \\
176(50,9)\end{array}$ & $\begin{array}{r}30(55,6) \\
170(49,1)\end{array}$ & 0,771 \\
\hline $\begin{array}{r}\text { Diabetes } \\
\text { - Sim } \\
\text { - Não }\end{array}$ & $\begin{array}{r}9(39,1) \\
191(50,7)\end{array}$ & $\begin{array}{r}14(60,9) \\
186(49,3)\end{array}$ & 0,283 \\
\hline $\begin{array}{l}\text { HTA } \\
\text { - Sim } \\
\text { - Não }\end{array}$ & $\begin{array}{r}8(27,6) \\
192(51,8)\end{array}$ & $\begin{array}{r}21(72,4) \\
179(48,2)\end{array}$ & 0,012 \\
\hline
\end{tabular}

IMC: Índice de massa corporal; HTA: Hipertensão arterial
Foram consideradas gestações de termo aquelas que apresentassem uma duração igual ou superior a 37 semanas. Reinternamento materno definiu-se como todo o internamento que tenha ocorrido no Serviço de Ginecologia/Obstetrícia no período de 60 dias após a alta hospitalar.

O protocolo do estudo foi submetido a avaliação pela Comissão de Ética da Administração Regional de Saúde do Centro, tendo obtido um parecer positivo e o estudo foi igualmente avaliado e aceite pela Direção do Serviço de Ginecologia/Obstetrícia do hospital em causa.

Foi criada uma base de dados no PASW® 18.0 e analisada a associação entre as variáveis e o tipo de parto, através da análise estatística bivariada, com cálculo do odds-ratio (OR) e aplicação do teste do qui-quadrado, adotando-se um nível de significância de 0,05 .

\section{RESULTADOS}

A amostra de 400 partos (200 cesarianas e 200 vaginais) estudados correspondeu a $17,4 \%$ dos 2.294 partos ocorridos no Hospital Santo André no ano de 2010.

Considerando os indicadores sociodemográficos maternos (Quadro I), constatou-se que as faixas etárias mais representativas na amostra de utentes, quer nas submetidas a cesariana, quer nas submetidas a parto vaginal, foram a dos 30-34 anos (respetivamente com $41,5 \%$ e $33,5 \%$ ) e a dos $25-29$ anos (com $29,0 \%$ versus $26,5 \%$ ). Quanto à escolaridade materna, salienta-se o facto de $81 \%$ das utentes submetidas a cesariana e $65 \%$ das submetidas a partos vaginais apresentarem escolaridade superior a 10 anos, sendo que $35,0 \%$ e $23,0 \%$, respetivamente, completaram o ensino superior.

Quarenta e cinco por cento das grávidas submetidas a cesariana e $37,5 \%$ 
daquelas cujo parto foi vaginal apresentavam IMC dentro da normalidade. Quanto aos hábitos tabágicos, $17,0 \%$ das utentes eram fumadoras $(15,0 \%$ submetidas a cesariana e $2,0 \%$ a parto vaginal).

Considerando os fatores reprodutivos pré-gestacionais, a percentagem de utentes multíparas foi superior entre as submetidas a parto vaginal $(52,5 \%)$. Utentes submetidas a cesariana apresentavam maior taxa de cesariana prévia $(28,5 \%$ comparativamente com $4,0 \%$ daquelas em que o parto foi vaginal). Quanto aos antecedentes patológicos maternos, a percentagem de diabetes e HTA foi superior nas utentes submetidas a cesariana $(7,0 \%$ e $10,5 \%$ versus $4,5 \%$ e $4,0 \%)$.

Os Quadros I,II e III comparam globalmente as utentes submetidas a cesariana e aquelas cujo parto foi vaginal.

Verificou-se que as utentes com escolaridade inferior a 4 anos $(n=4)$ apresentaram uma taxa de cesariana de $100 \%$. Nas restantes verificou-se que, quanto maior o nível de escolaridade, maior a taxa de cesarianas, atingindo esta última um máximo de $60,3 \%$ nas utentes que concluíram o ensino superior ( $p$ $<0,001)$. Utentes com escolaridade superior a 12 anos apresentaram um OR de 1,88 ( $p=0,004)$ (Quadro IV).

No que concerne ao IMC materno, desconhecia-se o seu valor em $4,0 \%$ dos casos. Apenas $45,0 \%$ das grávidas submetidas a cesariana e $37,5 \%$ daquelas cujo parto foi vaginal apresentavam IMC dentro da normalidade. A percentagem de utentes com excesso de peso e obesidade foi assim de $31,0 \%$ e $20,5 \%$ nas cesarianas e $39,5 \%$ e $22,5 \%$ nos vaginais. Das 392 utentes em que havia registo de IMC encontrou-se um OR para cesariana no IMC normal versus aumentado de 1,45, com um nível de significância de 0,073 (Quadro IV).

Quanto aos hábitos maternos, as utentes com hábitos tabágicos apresentaram uma taxa de cesariana superior à dos partos vaginais $(55,6 \%$ versus $44,4 \%)$, com um $O R$ de 1,29, contudo, esta associação não foi estatisticamente significativa ( $p=0,771)$ (Quadro IV).
Considerando os fatores reprodutivos pré-gestacionais, verificou-se que nas utentes multíparas a taxa de parto vaginal foi superior à de parto por cesariana, panorama inverso ao observado nas utentes nulíparas, que foram submetidas a maior número de cesarianas (57,0\% versus $43,0 \%$ ), com um OR de 1,88 e $p=0,002$ (Quadro IV).

Quanto à influência do tipo de parto prévio, detetou-se que as utentes com antecedentes de parto por cesariana apresentaram uma recorrência de cesariana de $87,7 \%$ (OR 9,57, $p<0,001$ ) (Quadro IV).

Em relação à vigilância da gravidez, detetou-se um efeito protetor sobre a ocorrência de cesariana para mães que fizeram a vigilância da gestação no setor público (OR 0,49, $p=0,002$ ) (Quadro IV).

Quanto aos antecedentes patológicos maternos, a percentagem de diabetes e HTA foi superior nas utentes submetidas a cesariana, $60,9 \%$ e $72,4 \%$ versus $39,1 \%$ e 27,6\%. A associação entre a diabetes e a realização de cesariana não foi estatisticamente significativa $(O R$ $1,59, p=0,283$ ), contrariamente à apresentada para a HTA (OR 2,82, $p=0,012)$ (Quadro IV). 
QUADRO III. Complicações do pós-parto e tipo de aleitamento materno à data da alta na amostra segundo o tipo de parto.

\begin{tabular}{l|r|r|r} 
Parâmetros avaliados & $\begin{array}{r}\text { Parto vaginal } \\
\mathrm{n}(\%)\end{array}$ & $\begin{array}{r}\text { Parto por cesariana } \\
\mathrm{n}(\%)\end{array}$ & $\begin{array}{c}\text { P-value } \\
\text { (Qui-Quadrado) }\end{array}$ \\
\hline $\begin{array}{l}\text { Aleitamento materno } \\
\text { exclusivo à data da alta }\end{array}$ & $179(53,0)$ & $159(47,0)$ & \\
- LM exclusivo & $4(19,0)$ & $17(81,0)$ & 0,005 \\
- LA & $14(41,5)$ & $24(58,5)$ & \\
- Misto & $1(20,0)$ & $4(80,0)$ & 0,177 \\
\hline $\begin{array}{l}\text { Reinternamento } \\
\text { - Sim }\end{array}$ & $199(50,4)$ & $196(49,6)$ & \\
- Não & $4(80,0)$ & $1(20,0)$ & 0,177 \\
\hline Hemorragia no pós-parto & $196(49,6)$ & $199(50,4)$ & \\
- Sim & $2(11,1)$ & $16(88,9)$ & 0,001 \\
- Não & $198(58,1)$ & $184(48,2)$ & \\
\hline Infeção no pós-parto & & & \\
- Sim & &
\end{tabular}

LM: Leite materno; LA: Leite artificial
Na presença de macrossomia fetal ou parto pré-termo, o tipo de parto mais prevalente foi a cesariana $(68,8 \%$ e $65,8 \%$, respetivamente), embora esta relação não seja estatisticamente significativa.

No que concerne aos reinternamentos, em 80,0\% das utentes reinternadas o parto ocorreu por cesariana (OR 4,06, $p=0,177$ ). Embora a taxa de hemorragia no pós-parto tenha sido inferior nas utentes submetidas a cesariana, esta associação não foi estatisticamente significativa $(O R$ $0,25, p=0,177)$. Quanto aos casos de infeção no período pós-parto, 88,9\% surgiram após realização de cesariana, com uma associação estatisticamente significativa $(O R 8,61, p=0,001)$ (Quadro IV).

\begin{tabular}{|c|c|c|c|}
\hline Parâmetros avaliados & OR (IC 95\%) & Intervalo confiança $95 \%$ & p-value \\
\hline Idade materna superior a 35 anos & 0,89 & $0,55-1,44$ & 0,623 \\
\hline Escolaridade superior a 12 anos & 1,88 & $1,21-2,92$ & 0,004 \\
\hline IMC normal & 1,45 & $0,97-2,16$ & 0,073 \\
\hline Hábitos tabágicos maternos & 1,29 & $0,73-2,30$ & 0,771 \\
\hline Nulípara & 1,88 & $1,26-2,81$ & 0,002 \\
\hline Cesariana anterior & 9,57 & $4,43-20,68$ & 0,0004 \\
\hline Vigilância no setor público & 0,49 & $0,33-0,74$ & 0,001 \\
\hline Antecedentes de diabetes & 1,59 & $0,68-3,78$ & 0,283 \\
\hline Antecedentes de HTA & 2,82 & $1,22-6,52$ & 0,012 \\
\hline Parto pré-termo & 2,06 & $1,02-4,14$ & 0,041 \\
\hline Macrossomia fetal & 2,27 & $0,77-6,66$ & 0,126 \\
\hline Reinternamento hospitalar & 4,06 & $0,45-36,66$ & 0,177 \\
\hline Infeção pós-parto & 8,61 & $1,95-37,95$ & 0,001 \\
\hline Hemorragia pós-parto & 0,25 & $0,27-2,22$ & 0,177 \\
\hline Sem aleitamento materno exclusivo à data de alta & 2,19 & $1,25-3,88$ & 0,006 \\
\hline
\end{tabular}

IMC: Índice de massa corporal; HTA: Hipertensão arterial 
Constatou-se ainda que, à data da alta hospitalar, $81,0 \%$ dos bebés sob aleitamento artificial e $58,5 \%$ dos sob aleitamento misto nasceram por cesariana, sendo que o $O R$ de não estar a fazer aleitamento materno exclusivo à data da alta, comparando as cesarianas com os partos vaginais, foi de 2,19 ( $p=0,006$ ) (Quadro IV).

As principais justificações apresentadas para a realização de cesariana foram, de acordo com o Quadro V, a incompatibilidade feto-pélvica, seguindo-se a suspeita de sofrimento fetal agudo e a indução falhada. A indução do trabalho de parto foi efetuada em $80(40,0 \%)$ das 200 utentes submetidas a cesariana, tendo sido infrutífera em $31,3 \%$ dos casos.

\section{DISCUSSÃO}

Apresentaram associação estatisticamente significativa com a realização de cesariana a elevada escolaridade, a nuliparidade, os antecedentes de cesariana prévia e a HTA. Tal não se verificou com os hábitos tabágicos, IMC materno, a idade materna, diabetes e macrossomia. O grupo das cesarianas apresentou um risco de infeção pós-parto 8 vezes superior e de aleitamento artificial na data da alta 2 vezes superior, associações estas também estatisticamente significativas. A taxa de reinternamento mostrou-se mais elevada nos partos por cesariana e a de hemorragia no pós-parto nos partos vaginais, associações estas, contudo, estatisticamente não significativas.

As principais justificações apresentadas para a realização de cesariana foram a incompatibilidade feto-pélvica, seguindo-se a suspeita de sofrimento fetal agudo e a indução falhada.

A baixa escolaridade materna assume um papel protetor para a cesariana, uma vez que a probabilidade de realização de cesariana foi 1,88 vezes superior em gestantes com escolaridade superior a 12 anos. Estes dados estão de acordo com os achados de outros autores. Um estudo efetuado com 540 puérperas, no Chile, constatou que as mulheres que apresentavam maior nível socioeconómico e eram vigiadas no setor privado apresentavam maior probabilidade de serem submetidas a cesariana, comparativamente com as vigiadas numa instituição pública. ${ }^{7,10}$ Para este facto foram apresentadas várias hipóteses explicativas, que poderão estar relacionadas com o serviço hospitalar, tipo de cuidados médicos oferecidos ou modo de participação da grávi-

\begin{tabular}{|c|c|}
\hline \multicolumn{2}{|c|}{$\begin{array}{l}\text { QUADRO V. Motivos apresentados para a realização de } \\
\text { cesariana. }\end{array}$} \\
\hline Motivo da cesariana & n (\%) \\
\hline Incompatibilidade feto-pélvica & $47(23,5)$ \\
\hline Sofrimento fetal agudo & $39(19,5)$ \\
\hline Indução falhada & $25(12,5)$ \\
\hline Cesariana anterior & $23(11,5)$ \\
\hline Eletiva por patologia materna & $22(11,0)$ \\
\hline Apresentação fetal anómala & $21(10,5)$ \\
\hline Distócia & $16(8,0)$ \\
\hline DPPNI & $3(1,5)$ \\
\hline Gravidez gemelar & $4(2,0)$ \\
\hline
\end{tabular}

DPPNI: Descolamento prematuro de placenta normalmente inserida

da no processo decisório do tipo de parto. ${ }^{10}$

No que diz respeito aos antecedentes verificou-se, à semelhança de outros estudos, que a nuliparidade apresentou associação significativa com um risco superior de cesariana, sendo que as nulíparas apresentaram uma probabilidade de parto por cesariana $1,88 \mathrm{ve}$ zes maior. ${ }^{8,12}$ As multíparas com parto prévio por cesariana, apresentam uma elevada taxa de recorrência da técnica $(87,7 \%)$, apresentando uma probabilidade 9,5 vezes superior de ter uma nova cesariana. De facto, um dos motivos que tem vindo a ser apresentado para a manutenção de elevadas taxas de cesarianas é a repetição da técnica. Estudos apontam que cerca de $30 \%$ das cesarianas são condicionadas pela existência de um parto anterior por cesariana. ${ }^{11}$ Muito embora novas guidelines recomendem a tentativa de parto vaginal após uma cesariana e tenham indicado que a prova de trabalho de parto, nestes casos, está associada a uma taxa de sucesso de parto vaginal entre 60 e $80 \%$, a recorrência de cesariana por cesariana prévia tem-se mantido elevada. ${ }^{11,13}$

No que diz respeito aos antecedentes patológicos maternos, no que se refere à HTA estes são congruentes com os dados encontrados na bibliografia, que referem a existência de um risco superior de realização de cesariana em gestantes com HTA. ${ }^{8}$ Neste caso, as grávidas hipertensas apresentaram um risco de parto 
por cesariana 2,8 vezes superior às que não apresentavam a patologia. Embora a diabetes seja uma das indicações para a prática de cesariana, muito devido à relação entre esta patologia materna e a macrossomia fetal, e apesar da prevalência de diabetes ter sido superior no grupo das cesarianas, não se verificou uma associação estatisticamente significativa entre estas duas variáveis. ${ }^{3}$

Relativamente às complicações no pós-parto, verificou-se um risco inferior de hemorragia e uma maior probabilidade de infeção e reinternamento no grupo de mulheres submetidas a cesariana. Com exceção dos dados referentes à taxa de hemorragia, os dados são congruentes com a literatura. Loverro et al, analisando a morbilidade febril pós-cirúrgica, referem que $5,14 \%$ dos casos cujo parto foi realizado por cesariana apresentaram complicação infeciosa, percentagem superior à observada após parto por via vaginal $(0,29 \%) \cdot{ }^{14} \mathrm{Um}$ estudo canadiano revelou a existência de um risco de infeção três vezes superior numa cesariana planeada comparativamente com um parto vaginal programado. ${ }^{15} \mathrm{Já}$ no que se refere ao risco de hemorragia, no estudo de Loverro et al foi avaliada a hemorragia de forma indireta, analisando a necessidade de transfusão de sangue no período intraparto e pós-parto, tendo-se verificado no período pós-parto uma proporção significativamente maior de casos transfundidos após cesarianas $(1,9 \%)$ quando comparados aos partos vaginais $(0,39 \%) .{ }^{14}$ Já no estudo realizado no Canadá, ainda que a cesariana planeada tenha estado associada a um risco superior de hemorragia com necessidade de realização de histerectomia, a percentagem de casos de hemorragia com necessidade de realização de transfusão sanguínea foi superior no grupo dos partos por via vaginal. ${ }^{15}$

Recém-nascidos cujo parto decorreu por cesariana apresentaram uma probabilidade de não fazerem aleitamento materno exclusivo à data da alta duas vezes superior. Estes dados, à semelhança dos dados encontrados noutros estudos, apontam a cesariana como uma barreira à implementação inicial e manutenção do aleitamento materno. ${ }^{5} \mathrm{O}$ parto vaginal está associado a uma maior probabilidade de aleitamento materno exclusivo aos 6 meses. ${ }^{16,17}$

No que concerne aos motivos apresentados para a realização de cesariana na amostra em causa, as prin- cipais justificações ostentadas foram, por ordem decrescente, a incompatibilidade feto-pélvica $(23,5 \%)$, o sofrimento fetal agudo (19,5\%), a indução de trabalho de parto falhada (12,5\%) e a existência de antecedentes de cesariana prévia (11,5\%). Estes dados são semelhantes aos encontrados num estudo levado a cabo na região norte do país, no qual os principais motivos registados foram a incompatibilidade feto-pélvica (23,6\%), o trabalho de parto estacionário $(20,7 \%)$, a suspeita de sofrimento fetal (20\%), a apresentação pélvica (14\%) e a tentativa frustrada de indução do trabalho de parto $(5,2 \%) .{ }^{9}$ Neste estudo, realizado no norte do país, não foi avaliado o impacto da existência de cesariana anterior. Como se verifica com base nestes resultados, a taxa de indução falhada na amostra em causa foi cerca do dobro da encontrada em estudos semelhantes. Segundo um estudo brasileiro publicado em 2011, para as gestações que atingem as 41 semanas e patologias obstétricas que coloquem em risco a vida materna ou fetal e que necessitem do término da gestação, deve iniciar-se a preparação do colo e indução do trabalho de parto. ${ }^{8}$ No entanto, a indução do trabalho de parto não está isenta de riscos, principalmente na ausência de colo favorável (trabalho de parto prolongado, superior a 24 horas; hiperestimulação uterina com baixa oxigenação fetal; rotura uterina; hemorragia pós-parto; corioamniotite; e necessidade de trabalho de parto instrumentalizado). ${ }^{8}$ No entanto, tem vindo a assistir-se à utilização da indução do trabalho de parto sem motivo médico, apenas como forma de programação do momento do nascimento, por conveniência da grávida e/ou da equipa de saúde. Nalguns países a indução do trabalho de parto é realizada em mais de $20 \%$ dos nascimentos, desconhecendo-se estes números nos hospitais públicos e privados nacionais. ${ }^{8}$

Uma das principais limitações deste estudo prende-se com o facto de ser retrospetivo, com base na consulta de processos clínicos, preenchidos por diferentes profissionais de saúde, não sendo possível excluir um viés de informação em relação a determinadas questões, por variabilidade interpessoal dos registos/medição. Não é possível, ainda, excluir um viés de seleção que, no entanto, foi minimizado pelo tamanho da amostra.

Apesar de se ter rejeitado a hipótese nula no que se refere às variáveis escolaridade, primiparidade, exis- 
tência de cesariana anterior, vigilância obstétrica no setor privado e antecedentes de HTA, não se pode excluir a existência de um erro aleatório tipo I, atendendo ao elevado número de hipóteses nulas consideradas. Atendendo a que o tamanho da amostra ficou aquém do calculado (200 casos versus os 252 necessários), não se pode igualmente excluir erros do tipo II, decorrentes da limitação do poder do estudo pelo tamanho amostral. Dado os resultados encontrados se referirem a uma amostra muito restrita, unicêntrica, estes não podem ser extrapolados para a população externa.

A elevada taxa de cesariana praticada em Portugal assume-se como um problema de saúde pública, que deverá ser alvo de uma intervenção multidisciplinar, com vista à sua redução. Tendo por base a experiência na região norte do país, o Ministério da Saúde criou, por despacho publicado no dia 5 de março de 2013, em Diário da República, a Comissão Nacional para a Redução da Taxa de Cesarianas, no âmbito da Direção-Geral da Saúde, que visa a criação de normas de orientação clínica e seus indicadores de monitorização, a formação profissional, a criação de um plano de educação para a saúde com criação de cursos para a parentalidade e a criação de um plano de auditorias internas/externas. A revisão sistemática que procedeu à análise deste tipo de medida considerou que este processo de auditoria e feedback apresenta uma boa efetividade. ${ }^{9}$

De futuro seria importante, ainda, avaliar a informação de que as grávidas dispõem relativamente aos benefícios do parto vaginal, quer para o bem-estar materno, quer para a saúde fetal e o seu desejo quanto ao tipo de parto.

Estudos revelam que a maioria das mulheres que preferem o parto por cesariana mudam de ideias após intervenções pré-natais e que os principais motivos para a solicitação de cesariana são o medo da dor, a ansiedade, o receio da perda de controlo e preocupação com lesão ou morte fetal. ${ }^{18,19}$ Enquanto médicos de família, temos o dever de informar as grávidas relativamente aos benefícios do parto vaginal, desmistificar os seus receios infundados, fornecendo-lhes conhecimentos que lhes permitam tomar uma decisão consciente e fundamentada.

\section{REFERÊNCIAS BIBLIOGRÁFICAS}

1. World Health Organization. The World Health Report 2005. Geneva:
WHO; 2005.

2. Patah LE, Malik AM. Models of childbirth care and cesareans rates in different countries. Rev Saúde Pública 2011 Feb; 45 (1): 185-94.

3. Campos I. Sistematização das indicações para cesariana. In: Campos DA, Montenegro N, Rodrigues T, editores. Protocolos de Medicina. Lisboa: Lidel; 2008.

4. Leeman LM, Plante LA. Patient-choice vaginal delivery. Ann Fam Med 2006 May-Jun; 4 (3): 265-8.

5. Rowe-Murray HJ, Fisher JR. Baby friendly hospital practices: cesarean section is a persistent barrier to early initiation of breastfeeding. Birth 2002 Jun; 29 (2): 124-31.

6. Brennan DJ, Robson MS, Murphy M, O'Herlihy C. Comparative analysis of international cesarean delivery rates using 10-group classification identities significant variation in spontaneous labour. Am J Obstet Gynecol 2009 Sep; 201 (3): 308.e1-308.e8.

7. Mendoza-Sassi RA, Cesar JA, Silva PR, Denardin G, Rodrigues MM. Risk factors for cesarean section by category of health service. Rev Saúde Pública 2010 Feb; 44 (1): 80-9.

8. Silveira DS Santos IS. Fatores associados à cesariana entre mulheres de baixa renda em Pelotas, Rio Grande do Sul, Brasil. Cad Saúde Pública 2004; 20 (2): 231-41.

9. Campos D, Furtado J, Crisóstomo M, Carrapato R, Cunha E, Conceição M. Medidas para reduzir a taxa de cesarianas na Região Norte de Portugal. Ministério da Saúde - ARS Norte. Disponível em: http://portal.arsnorte.min-saude.pt/portal/page/portal/ARSNorte/Conte\% C3\%BAdos/GRP/Ficheiros/Cesarianas/Relatorio_Taxas_Cesarianas.pdf [acedido em 26/10/2011].

10. Faisal-Cury A, Menezes PR. Factors associated with preference for cesarean delivery. Rev Saúde Pública 2006 Apr; 40 (2): 226-32.

11. Gomes F, Marques M, Ferreira J. Parto vaginal em grávidas com cesariana anterior. In: Campos DA, Montenegro N, Rodrigues T. Protocolos de Medicina Materno-Fetal. Lisboa: Lidel; 2008.

12. Villar J, Valladares E, Wojdyla D, Zavaleta N, Carroli G, Velazco A, et al. Caesarean delivery rates and pregnancy outcomes: the $2005 \mathrm{WHO}$ global survey on maternal and perinatal health in Latin America. Lancet 2006 Jun 3; 367 (9525): 1819-29.

13. Guise JM, Eden K, Emeis C, Denman MA, Marshall N, Fu R, et al. Vaginal Birth after Cesarean: new insights. Evidence Report/Technology Assessment, nr 191. Rockville, MD: Agency for Healthcare Research and Quality; 2010.

14. Nomura RM, Alves EA, Zugaib M. Maternal complications associated with type of delivery in a university hospital. Rev Saúde Pública 2004 Feb; 38 (1): 9-15.

15. Liu S, Liston RM, Joseph KS, Heaman M, Sauve R, Kramer MS; Maternal Health Study Group of the Canadian Perinatal Surveillance System. Maternal mortality and severe morbidity associated with low-risk planned cesarean delivery versus planned vaginal delivery at term. CMAJ 2007 Feb 13; 176 (4): 455-60.

16. Al-Sahab B, Lanes A, Feldman M, Tamim H. Prevalence and predictors of 6-month exclusive breastfeeding among Canadian women: a national survey. BMC Pediatrics 2010 Apr 8; 10: 20-30.

17. Vieira TO, Vieira GO, Giugliani ER, Mendes CM, Martins CC, Silva LR. Determinants of breastfeeding initiation within the first hour of life in a Brazilian population: cross-sectional study. BMC Public Health 2010 
Dec 9; 10: 760.

18. Dias MA, Domingues RM, Pereira AP, Fonseca SC, da Gama SG, Theme Filha MM, et al. Trajetória das mulheres na definição pelo parto cesáreo: estudo de caso em duas unidades do sistema de saúde suplementar do estado do Rio de Janeiro. Ciên Saúde Colet 2008 sep-out; 13 (5): 1521-34.

19. Haddad SE, Cececatti JG. Estratégias dirigidas aos profissionais para a redução das cesáreas desnecessárias no Brasil. Rev Brasileira Ginecol Obstetr 2011 mai; 33 (5): 252-62.

\section{DECLARAÇÃO DE INTERESSES}

A autora declara não ter conflitos de interesse nem ter recebido qualquer financiamento para o presente estudo.

\section{ENDEREÇO PARA CORRESPONDÊNCIA}

Ana Rita Oliveira

Rua Dr. José Oliveira Baptista, $n^{\circ}$ 6, $2^{\circ}$ direito, 2300-491 Tomar

Email: rita-olive@hotmail.com

Recebido em 03/09/2012

Aceite para publicação em 29/04/2013

\section{ABSTRACT}

\section{DETERMINANTS FOR THE PRACTICE OF CAESARIAN SECTION: A CASE-CONTROL STUDY}

Objectives: To determine if years of maternal education, body mass index (BMI), smoking, nulliparity, follow-up of pregnancy in the private sector, previous cesarean section (C-section), diabetes, hypertension and large birth weight are associated with increased risk for having a C-section, to test the association between C-section and postpartum complications (hemorrhage, infection and readmission), the association between $\mathrm{C}$-section and breastfeeding at discharge and to describe the indications for C-section.

Study design: Case control study

Setting: Leiria-Pombal Hospital Centre.

Participants: A random, stratified, non-proportional sample of 400 women (200 with C-section and 200 with vaginal delivery) giving birth in the Leiria-Pombal Hospital in 2010 was selected.

Methods: Tests of association between variables and the type of delivery was done by bivariate analysis with calculation of the odds-ratio, using the Chi-square test with significance set at the 0.05 level.

Results: The rate of $C$-sections increased the with the level of maternal education $(p<0,001)$. In the $\mathrm{C}$-section group, the rate of nulliparity was $57,0 \%(p=0,002)$. Among multiparous women undergoing a C-section $87,7 \%$ had a previous $C$-section $(p<0,001)$. In the $C$-section group a higher rate was found of hypertension $(72,4 \%, p=0,012)$ and of follow-up of pregnancy in the private sector $(56,9 \%, p=0,002)$. Infants receiving formula $(81 \%)$ and infants receiving mixed formula and breast feeding at hospital discharge $(58,5 \%)$ were more likely to have been delivered by $C$-section $(p=0,005)$. When infection complicated delivery, this was most often after $C$-section $(88,9 \%, p=0,001)$. Statistical significant associations were not found between $\mathrm{C}$-section and BMI, smoking, large birth weight, and diabetes.

Conclusions: Higher maternal educational level, nulliparity, follow-up of pregnancy in the private sector, previous C-section, hypertension, the use of formula at discharge, and post-partum infection were association with C-section.

Mesh Terms: Cesarean Section, Obstetric Delivery, Prevalence. 